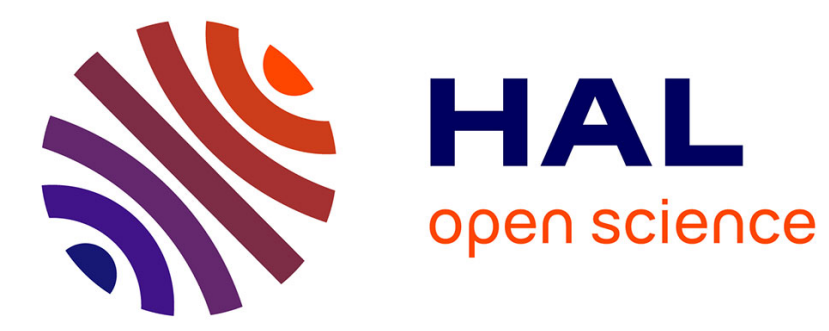

\title{
Design rules for nonlinear spectral compression in optical fibers
}

Christophe Finot, Sonia Boscolo

\section{To cite this version:}

Christophe Finot, Sonia Boscolo. Design rules for nonlinear spectral compression in optical fibers. Journal of the Optical Society of America B, 2016, 33 (4), pp.760-767. 10.1364/JOSAB.33.000760 . hal-01275273

\section{HAL Id: hal-01275273 \\ https://hal.science/hal-01275273}

Submitted on 17 Feb 2016

HAL is a multi-disciplinary open access archive for the deposit and dissemination of scientific research documents, whether they are published or not. The documents may come from teaching and research institutions in France or abroad, or from public or private research centers.
L'archive ouverte pluridisciplinaire HAL, est destinée au dépôt et à la diffusion de documents scientifiques de niveau recherche, publiés ou non, émanant des établissements d'enseignement et de recherche français ou étrangers, des laboratoires publics ou privés. 


\title{
Design rules for nonlinear spectral compression in optical fibers
}

\author{
ChRISTOPHE FINOT, ${ }^{1, *}$ SONIA BOSCOLO ${ }^{2}$ \\ ${ }^{1}$ Laboratoire Interdisciplinaire Carnot de Bourgogne, UMR 6303 CNRS-Université de Bourgogne/Franche-Comté, 9 avenue Alain Savary, BP \\ 47870, 21078, Dijon Cedex, France \\ ${ }^{2}$ Aston Institute of Photonic Technologies, School of Engineering and Applied Science, Aston University, Birmingham B4 7ET, United Kingdom \\ *Corresponding author: christophe.finot@u-bourgogne.fr
}

Received XX Month XXXX; revised XX Month, XXXX; accepted XX Month XXXX; posted XX Month XXXX (Doc. ID XXXXX); published XX Month XXXX

\begin{abstract}
We present comprehensive design rules to optimize the process of spectral compression arising from nonlinear pulse propagation in an optical fiber. Extensive numerical simulations are used to predict the performance characteristics of the process as well as to identify the optimal operational conditions within the space of system parameters. It is shown that the group-velocity dispersion of the fiber is not detrimental and, in fact, helps achieve optimum compression. We also demonstrate that near-transform-limited rectangular and parabolic pulses can be generated in the region of optimum compression.
\end{abstract}

OCIS codes: (060.4370) Nonlinear optics, fibers; (060.7140) Ultrafast processes in fibers; (060.5530) Pulse propagation and temporal solitons.

\section{INTRODUCTION}

The optical nonlinearity in a fiber is ordinarily associated with spectral broadening of an ultrashort optical pulse. In certain regimes, however, the fiber nonlinearity can induce the opposite effect, resulting in spectral compression of a pulse [1]. For example, a fundamental soliton propagating in an anomalous dispersion-increasing fiber can experience narrowing of its spectral width as a result of its adiabatic adaptation to the slowly varying fiber dispersion [2]. The working principle is the reverse operation of the well-known adiabatic soliton temporal compression in a dispersion-decreasing fiber [3]. Ultrashort laser pulses can be spectrally compressed in the regime of soliton selffrequency shift induced by the Raman effect in a highly nonlinear fiber [4]. Owing to the anomalous dispersion of the highly nonlinear fiber, laser pulses evolve toward solitons and experience a continuous frequency downshift. A lowering frequency and increasing dispersion of a red-shifting soliton dictate spectral narrowing. Another way to realize spectral compression relies on the self-phase modulation (SPM) of a pulse with an initial negative frequency modulation (chirp) [5-7]. Indeed, the intensity-dependent nonlinear phase shift induced by SPM results, in silica, in a frequency downshift in the leading edge of the pulse and an upshift in the trailing edge. Thus the effect of SPM is dependent on the sign of the initial chirp. Negatively chirped pulses, where the long and the short wavelengths are in the trailing and the leading edges, respectively, are spectrally compressed, since both the long and the short wavelengths are shifted toward the center wavelength. This method of spectral compression has been implemented using standard single-mode fibers [8], optical gain fibers [9-12], and photonic crystal fibers [13-15], and is suitable for a very large range of wavelengths including Ti:sapphire wavelengths $[8,13]$, the widely used $1-\mu \mathrm{m}[9,11]$ and $1.55-\mu \mathrm{m}[16]$ windows and the emerging $2-\mu \mathrm{m}$ band [12]. However, most of the discussions and demonstrations using this approach have been carried out in the nonlinearity-dominant regime of propagation in which the dispersion of the fiber is of little importance, as the dispersion and its interplay with SPM are commonly believed to ultimately limit the spectral compression performance [13]. The role of group-velocity dispersion (GVD) in the spectral compression process has been discussed in [7, $13,17]$.

In this paper, we present an accurate analysis of the impact of normal GVD on the spectral compression by SPM of negatively chirped pulses in a fiber, which extends and generalizes the prior works of [7, $13,17]$ in several respects. Relevantly, the studies reported in $[17,18]$ showed that the GVD can change substantially the physical pattern of the spectral compression process: the combined action of GVD and SPM can result in a deformation of the temporal profile of the pulse tending to acquire a rectangular shape while complete compensation of the pulse chirp occurs. Here, we provide a thorough characterization of the process based on extensive numerical simulations. The optimal operational conditions are identified in terms of normalized input pulse power and fiber length for varying initial stretching ratio, which enables us to provide general design rules for spectral-compression fiber schemes over the parameter space that is typically accessible experimentally. Quite surprisingly, our results show that the fiber dispersion rather enhances the quality of the compression compared to the purely nonlinear regime. We also show that initially Gaussian pulses with a linear negative chirp experiencing spectral narrowing in the fiber can result in near-transform-limited rectangular or parabolic shaped pulses in the region of optimum compression, in accordance with previous results $[17,18]$. Firstly, we introduce the situation being studied and the metrics used to quantify the performance of the compression process. Next we recall the results that can be obtained in the purely nonlinear case, and then we describe the effects of normal GVD. Finally, we discuss the influence of the initial stretching ratio and pulse shape on the performances. 


\section{SITUATION UNDER INVESTIGATION}

For the purpose of illustration, we use both transform-limited pulses with a Gaussian intensity profile $\psi_{0}(t)=\sqrt{P_{0}} \exp \left(-t^{2} / 2 T_{0}^{2}\right)$ and an hyperbolic secant profile $\psi_{0}(t)=\sqrt{P_{0}} \operatorname{sech}\left(t / T_{0}\right)$ as initial conditions for our study. The parameters $T_{0}$ and $P_{0}$ are a characteristic temporal value and the peak power of the initial pulse, respectively; $T_{0}$ can be related to the full-width at half-maximum (FWHM) pulse duration as $T_{\mathrm{FWHM}}=K_{1} T_{0}$, where $K_{1}=1.665$ and $K_{1}=1.763$ for the Gaussian and hyperbolic secant pulses, respectively. The linear negative temporal chirp required for spectral compression to take place can be imprinted onto the initial pulse by stretching the pulse in the temporal domain in an anomalously dispersive medium, such as a pair of diffraction gratings [10,13], a prism pair [6, 8], a fiber Bragg grating, or a segment of hollow core [11] or standard [16] fiber with anomalous GVD (and very low nonlinearity). As a result of GVD (described by Eq. (1) with $\gamma=0$ ), the different spectral components of the pulse acquire a frequency dependent delay. Even though such phase changes do not affect the pulse spectrum, they lead to temporal broadening of the pulse and a time dependence of the pulse phase. If $A$ denotes the temporal broadening factor, the peak power of the pulse will drop by the same factor $\left(P_{1}=P_{0} / A\right)$ owing to the conservation of energy (in the absence of losses). In the far-field regime (i.e., when $A>>1$ ), the temporal phase becomes parabolic, so that the pulse envelope at the exit of the dispersive element takes the form $\psi(0, t)=\left|\psi_{1}(t)\right| \exp \left(-i b t^{2}\right)$, where $\left|\psi_{1}\right|$ is the stretched amplitude profile and $b<0$ is the chirp parameter. This negatively linearly chirped pulse is then spectrally compressed in a nonlinear fiber with normal GVD. Pulse propagation in the fiber can be described by the standard nonlinear Schrödinger equation (NLSE) [19] :

$$
i \frac{\partial \psi}{\partial z}-\frac{1}{2} \beta_{2} \frac{\partial^{2} \psi}{\partial t^{2}}+\gamma|\psi|^{2} \psi=0,
$$

where $z$ is the propagation distance, $t$ is the reduced time, $\beta_{2}$ is the GVD parameter, and $\gamma$ is the coefficient of cubic nonlinearity of the fiber. For the sake of simplicity, we do not include the effect of fiber loss, or higher-order linear or nonlinear effects in our model. Note that neglecting the effect of third-order dispersion is well suited to describe the evolution of a pulse undergoing progressive spectral narrowing in the fiber.

The narrowing of the pulse spectrum is quantified with the spectral compression factor $C$ defined as the ratio of the spectral FWHM width at some distance in the fiber and the entrance of the fiber. In order to assess the quality of the spectral compression, we use the Strehl ratio $S$ defined as the ratio of the maximum spectral brilliance of the actual pulse to the spectral brilliance obtained assuming a flat temporal phase of the pulse [16]. Therefore, $S$ is comprised between 0 and 1 , with 1 defining an ideal compression. We also characterize the temporal shape of the pulse by means of the excess kurtosis parameter $K[20$, 21] and the parameter of misfit $M$ between the pulse intensity profile and a parabolic fit $\left|\psi_{p}\right|^{2}$ of the same energy [22]:

$$
M^{2}=\int\left[|\psi(t)|^{2}-\left|\psi_{p}(t)\right|^{2}\right]^{2} d t / \int|\psi(t)|^{4} d t .
$$

\section{PULSE EVOLUTION IN A PURELY NONLINEAR MEDIUM}

The effects of SPM on a pulse propagating in a purely nonlinear medium are studied by setting $\beta_{2}=0$ in Eq. (1). In this case, the nonlinear term in (1) imposes a time-dependent phase on the pulse so that after a propagation length $z$ :

$$
\psi(z, t)=e^{i \gamma|\psi(0, t)|^{2} z} \psi(0, t)=e^{i \gamma\left|\psi_{1}(t)\right|^{2} z-i b t^{2}}\left|\psi_{1}(t)\right| .
$$

In general an input pulse with a negative linear chirp $\psi(0, t)$ cannot be spectrally compressed to the Fourier transform limit because the instantaneous frequency $\delta v(t)=-d \phi / d t=-\gamma d\left(\left|\psi_{1}\right|^{2}\right) / d t z+$ $2 b t$ cannot in general be made equal to zero for all times. However, if we approximate the initial Gaussian or hyperbolic secant shape $\left|\psi_{1}\right|^{2}$ by a parabola in the central region of the pulse, the resulting instantaneous frequency near the pulse center can be expressed as:

$$
\delta v(t)=-\frac{d}{d t} \phi(t)=2\left(\frac{\gamma P_{0} z}{A^{3} T_{0}^{2}}+b\right) t
$$

and, thus, yields zero for all $t$ at the propagation distance $z_{1} / L_{N L}=$ $-b A^{3} T_{0}^{2}$, where $L_{N L}=1 /\left(\gamma P_{0}\right)$ is the nonlinear length associated with the pulse at the input to the system. In the far-field conditions when $A \gg>1$, we can use the following empirical relationship between the broadening factor $A$ and the chirp coefficient $b$ :

$$
b \approx-\frac{\pi}{A} \frac{K_{2}}{T_{F W H M}^{2}}=-\frac{\pi}{A} \frac{K_{2}}{K_{1}^{2} T_{0}^{2}}
$$

where $K_{2}$ is the time-bandwidth product (TBP) of the initial transformlimited pulse $\psi_{0}$. This leads to a simple approximate expression for $z_{1}$ :

$$
z_{1} / L_{N L} \approx \pi \frac{K_{2}}{K_{1}^{2}} A^{2} .
$$
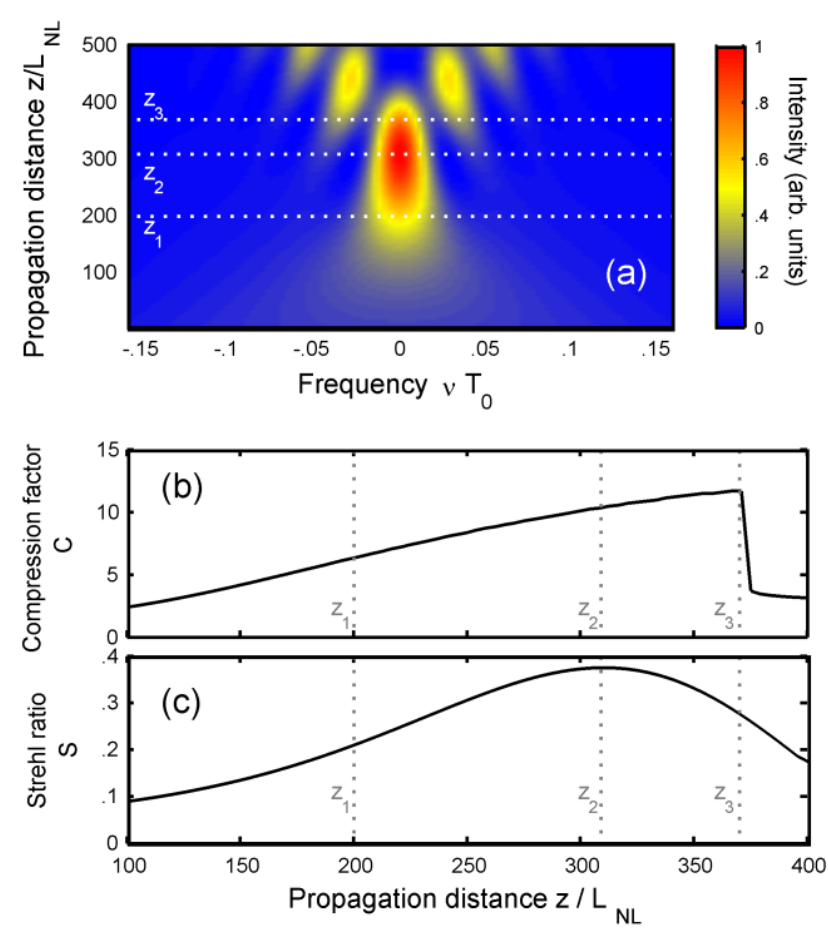

Fig. 1. Longitudinal evolution of: (a) the spectral intensity profile, (b) the spectral compression factor, and (c) the Strehl ratio for an initial Gaussian pulse with the stretching factor $A=20$ propagating in a purely nonlinear fiber.

An example of the evolution of an initial Gaussian pulse along the nonlinear fiber is presented in Fig. 1 for the initial stretching factor $A=$ 20. Significant spectral compression occurs in the fiber, followed by splitting of the pulse spectrum [panel (a)]. The evolutions of parameters $C$ and $S$ in panels (b) and (c), respectively, reveal that the propagation distance at which the spectral compression factor reaches its maximum value (denoted by $Z_{3}$ ) differs from the distance of optimum compression in terms of Strehl ratio (denoted by $z_{2}$ ), as well as from the distance $z_{1}$ of chirp cancellation near the pulse center given by Eq. (6). We can also infer from Fig. 1 that the highest brilliance in the central region of the pulse spectrum is achieved at the point of maximum Strehl ratio. At $z_{2}$, the spectral brilliance has increased by a factor of more than 6 compared to the input spectrum, and the gain in spectral brilliance is more than $60 \%$ compared with the propagation distance predicted by Eq. (6), whereas the FWHM spectral width of the pulse is only $12 \%$ larger than that at the point of maximal compression. After $z_{3}$, the compression factor $C$ drops abruptly due to 
an increase of the intensity level of spectral satellites above the $-3 \mathrm{~dB}$ threshold used for the computation of $C$. The pulse spectrum splits up shortly after $z 3$.

The characterizations of the pulse at the different operational distances in the fiber are given in Fig. 2. From panel (a) we can see that at the distance $z_{1}$ foretold by Eq. (6) the pulse spectrum is effectively conveniently compressed, and the spectral profile does not display any oscillations, which is in agreement with the monotonic temporal variation of the frequency chirp at this distance [panel (b)]: the chirp continuously decreases across the pulse, and has a point of inflexion at the pulse center. At the other operational distances, the spectrum features an oscillating structure, which results from interference between different pulse parts having the same instantaneous frequency. This is confirmed by the non-monotonic temporal variation of the chirp. Such a constructive interaction can have a beneficial impact on the spectral compression process as it can increase the peak spectral intensity of the pulse, as shown by the pulse spectrum obtained at the optimum compression point in terms of Strehl ratio $z_{2}$. But it can also induce strong and detrimental oscillations in the wings of the pulse and concomitant strong side lobes in the pulse spectrum, as it appears from the spectrum at the point of maximum compression factor $Z_{3}$. On the other hand, at $Z_{1}$, while the spectrum narrowing is less in terms of FWHM spectral width, the spectrum exhibits significantly lower substructures. We note that this scenario is similar to the situation when the $B$-integral accumulation in chirped pulse amplification systems is not exactly compensated for: as a result of the distortion of the linear chirp by SPM, temporal structure unavoidably appears in the recompressed pulse, even for relatively small values of $B$ [23]. The presence of residual pedestals in the compressed spectrum could be avoided by taking advantage of the strictly linear chirp induced by the SPM of a parabolic-shaped input pulse $[15,16]$.
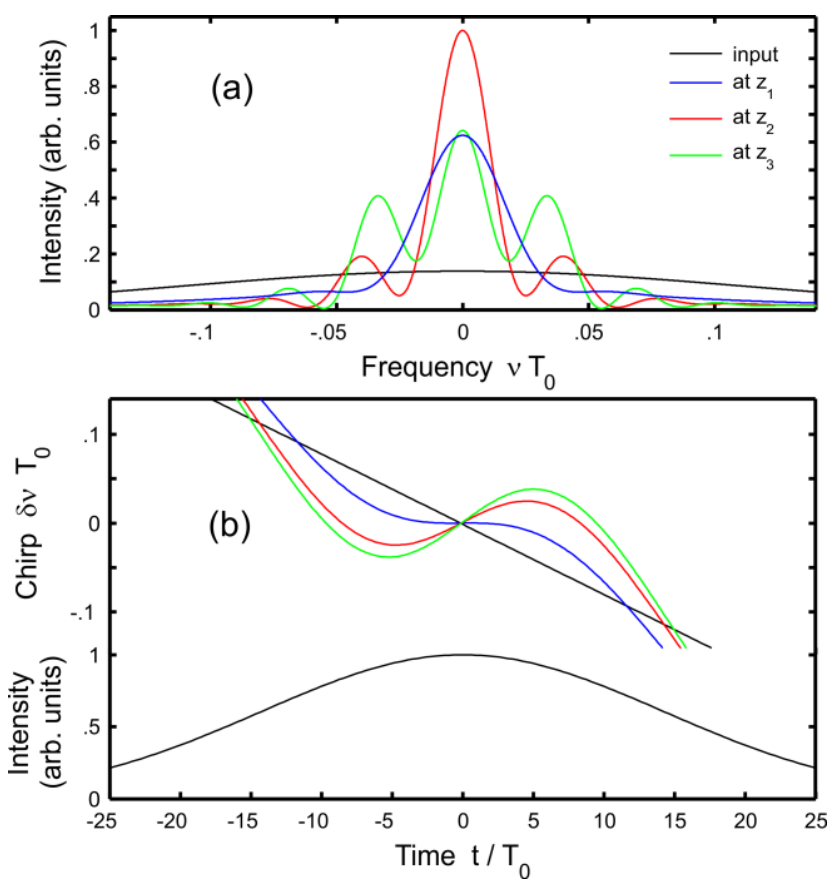

Fig. 2. (a) Spectral intensity profile, and (b) temporal intensity and chirp profiles of an initial Gaussian pulse with the stretching factor $A=$ 20 at different propagation distances in a purely nonlinear fiber: the point $z_{1}$ predicted by Eq. 6 (blue), the point $z_{2}$ of maximum Strehl ratio (red), and the point $z_{3}$ of maximum spectral compression factor (green). Also shown are the initial pulse profiles (black).
In the light of these considerations, we can conclude that the Strehl ratio plays a major role in determining the optimum working parameters for the spectral compression process. Indeed, the point of maximum $C$ brings about relatively strong spectral side lobes and low peak spectral intensity, and the simple guidelines provided by Eq. (4) (or Eq. (6)) are not accurate enough except for the case of a parabolic input pulse. It is also worth noting that the TBP, which is commonly used for indicating how close a pulse is to the transform limit, may be a misleading metric: for example, at the propagation distance $\mathrm{z}_{2}$ the TBP is 0.20 , which is well below the well-known value of a bandwidthlimited Gaussian-shaped pulse.

We also studied the effect of the initial stretching factor $A$ on the pulse evolution. In Fig. 3, we summarize the results obtained for Gaussian and hyperbolic secant initial conditions and using stretching values that are typical of experimental realizations [16]. It is seen that a larger $A$ results in a larger maximum compression factor. However, due to imperfect spectral compression, the maximum compression factor reaches only approximately half of the value $(C=A)$ that it would attain in the case of a Fourier transform-limited process. Furthermore, the overall quality of the process deteriorates with increasing $A$, as indicated by the decrease of the Strehl ratio. These results suggest a tradeoff between the degree of spectral narrowing and the growth of spectral satellites. A larger $A$ also leads to a longer optimum propagation distance in terms of Strehl ratio $z_{2}$, with the variation of $z_{2}$ with $A$ exhibiting the parabolic growth suggested by Eq. (6). The guidelines that can be drawn from these numerical results are found in qualitative agreement with the experiments on the spectral compression of picosecond pulses in a highly nonlinear fiber at telecommunication wavelengths reported in[16]. We can also see from Fig. 3 that the initial Gaussian pulse overall outperforms the hyperbolic secant pulse.

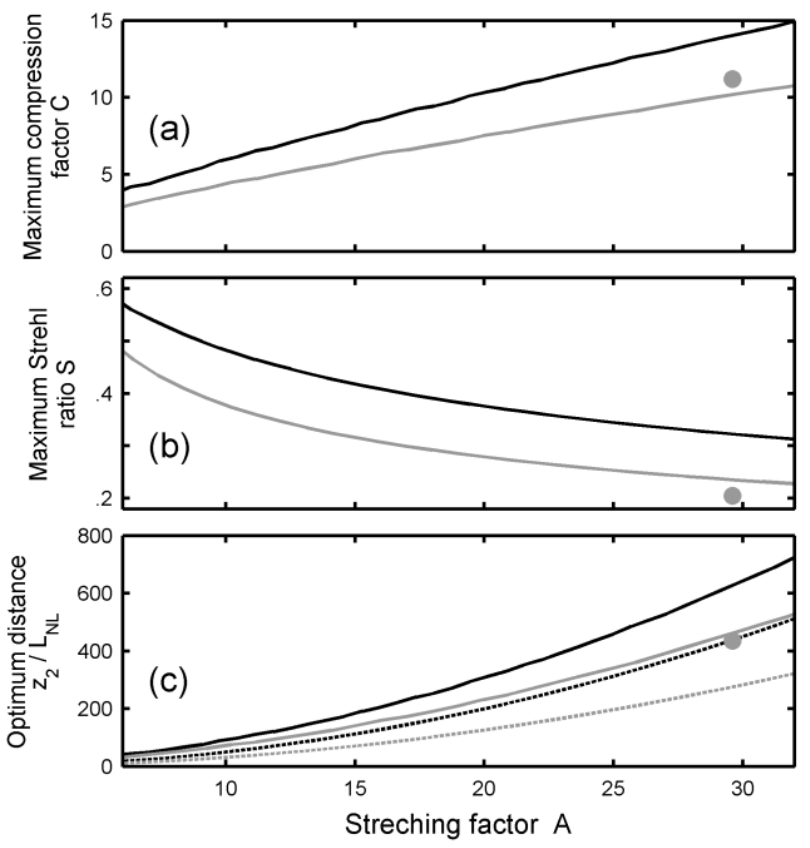

Fig. 3. Dependencies of: (a) the maximum spectral compression factor, (b) the maximum Strehl ratio, and (c) the optimum propagation distance in terms of Strehl ratio on the stretching factor $A$ for initial Gaussian (black) and hyperbolic secant (grey) pulses propagating in a purely nonlinear fiber. The predictions from Eq. (6) for the distance of approximate chirp cancellation are also shown for comparison (dashed lines). The grey circles represent the experimental results of [16] for an initial hyperbolic secant pulse. 


\section{PULSE EVOLUTION IN A NONLINEAR DISPERSIVE FIBER}

In this section, we perform a detailed analysis of the spectral compression process taking into account the influence of GVD.

\section{A. Normalization of the problem}

To simplify the analysis, we normalize the NLSE (1) by introducing the normalized distance variable $\xi=z / L_{D}$ and the parameter $N$ defined as $N^{2}=L_{D} / L_{N L}$, where $L_{D}=T_{0}^{2} / \beta_{2}$ is the dispersion length associated with the pulse at the input to the system. This way, for a given value of the initial stretching factor $A$, finding the best system parameters results in a two-dimensional optimization problem in the plane $(\xi, N)$. It is worth noting here that a change of $T_{0}$ implies a change of the dispersion length of the linear pre-chirping element, thereby of the stretching factor $A$. Therefore, in such case parameter $A$ should be correspondingly adjusted in order to interpret the predictions based on the scaling laws from the NLSE correctly. In this paper, we limit the discussion to normally dispersive fibers as we found that in the anomalous dispersion regime of a fiber the trends and performances are similar to those of the pure SPM configuration. Indeed, in the anomalous GVD region the optimal operational conditions are obtained for the highest input peak power, leading to the shortest propagation length and a nonlinearity-dominant regime of propagation.

\section{B. Evolution of the spectral characteristics}

The maps presented in Fig. 4 summarize the evolution of the spectral compression factor and the Strehl ratio versus $\xi$ and $N$ for an initial Gaussian pulse with the stretching factor $A=20$. Note that the computation of the Strehl ratio takes into account the actual pulse temporal profile and, thus, the evolution of $S$ carries information on the corresponding variation of the temporal profile. We can see from Fig. 4 that similarly to the purely nonlinear case, the combination of system parameters leading to maximal compression differs from the point of maximum Strehl ratio (indicated by a plus sign marker). It is also seen that there are two areas of operational conditions bearing high $S$ values. However, only area (1) corresponds to an effective significant spectral compression and, thus, we will focus our attention on this area. By contrast, area (2), obtained for longer propagation distances and lower input peak powers, supports a poor compression. This area can be partly understood in the framework of the weak-nonlinearity scenario analyzed in [7].

The pulse spectra obtained for different sets of system parameters are plotted in Fig. 5. We can see that at the point of optimum compression in terms of Strehl ratio, $\left(\xi_{\text {opt }}=13.6, N_{\text {opt }}=4.4\right)$, the central lobe of the spectrum is only slightly wider than that obtained at the point of maximal compression, $(\xi=6.8, N=7.1)$, while the intensity level of spectral satellites is reduced by more than twice. Notwithstanding, the quality of the compression is still degraded compared to the ideal compression of the initial pulse (i.e., before the actual propagation in the fiber causes deformations of the temporal waveform). Furthermore, Fig. 6 highlights that the GVD significantly change the evolution of the pulse spectrum in the fiber: contrary to the pure SPM case (Fig. 1(a)), the spectrum does not split into multiple sidebands after the stage of optimum compression.
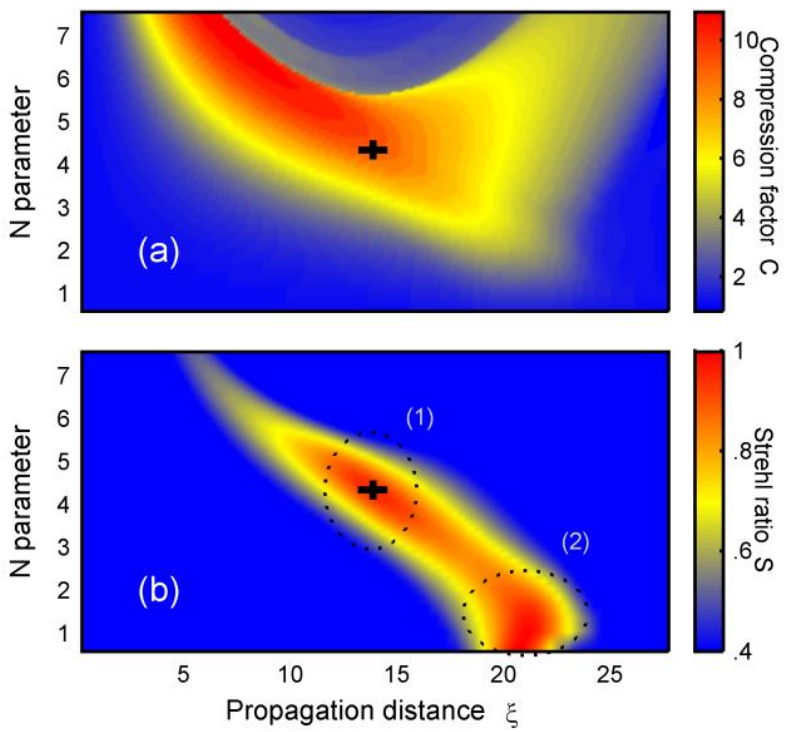

Fig. 4. Evolution of: (a) the spectral compression factor and (b) the Strehl ratio versus $\xi$ and $N$ in a nonlinear dispersive fiber for an initial Gaussian pulse with the stretching factor $A=20$. The sign plus denotes the point of maximum Strehl ratio.
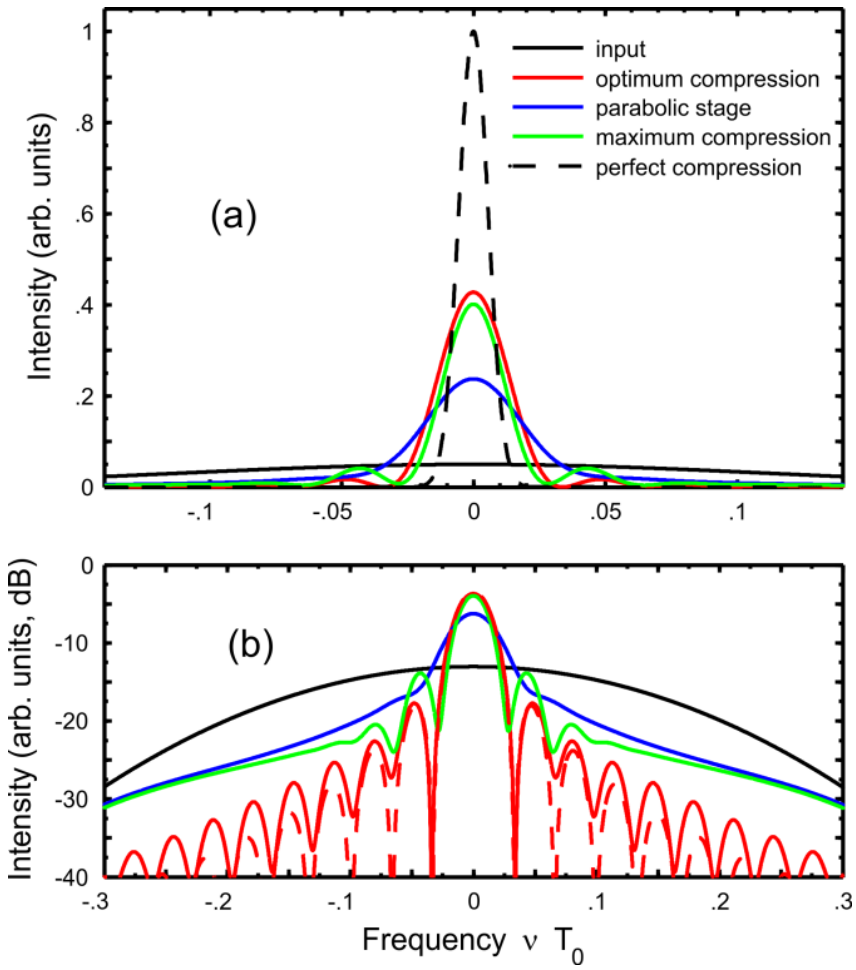

Fig. 5. Spectral intensity profiles of an initial Gaussian pulse with the stretching factor $A=20$ at different points in the plane $(\xi, N)$, plotted on (a) linear and (b) logarithmic scales. The spectrum of a pulse with the optimum power parameter $N_{\text {opt }}$ at the entrance of the fiber (solid black) is compared with the spectrum at the optimum compression point ( $\xi_{\text {opt, }} N_{\text {opt}}$ ) (solid red) and at the point of maximal compression (solid green). Also shown are the spectra that would result from the spectral compression of the initial pulse with $N_{\text {opt }}$ before actual propagation in the fiber (dashed black) and of the pulse at ( $\left.\xi_{\text {opt, }}, N_{\text {opt }}\right)$ after perfect propagation (dashed red), and the spectrum of the initial pulse with $N_{\text {opt }}$ at the stage where the temporal profile is close to a parabola (solid blue). 


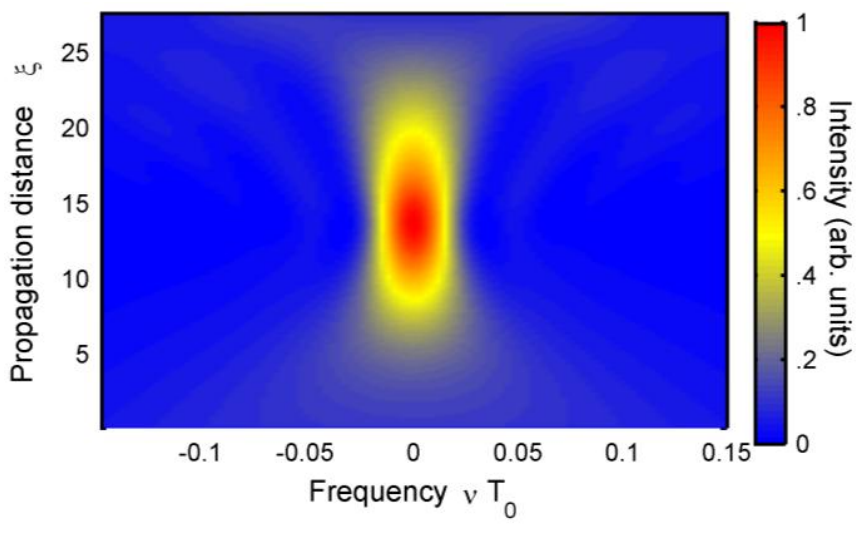

Fig. 6. Longitudinal evolution of the spectral intensity profile of an initial Gaussian pulse with the stretching factor $A=20$ and the optimum power parameter $N_{\text {opt. }}$

\section{Evolution of the temporal characteristics}

Unlike the purely nonlinear regime where the temporal profile of the pulse remains unchanged upon propagation, the combined action of GVD and SPM results in significant modifications of the pulse temporal characteristics. The evolution of the excess kurtosis parameter and the misfit parameter to a parabolic temporal shape versus $\xi$ and $N$ are summarized in the maps of Fig. 7. We can see that the excess kurtosis strongly evolves upon propagation: for the range of initial powers of interest ( $N$ boundary of region 1 ), it progressively decreases and reaches negative values. This indicates a flattening of the central part of the pulse [20], consistently with the usual trends observed in the regime of normal dispersion in the presence of strong nonlinearity [24, 25]. Note that for the powers within the characteristic range of region 2 , the kurtosis evolution is rather different in that it bears an increase of the peakedness of the pulse, suggesting that this region is better suited for the observation of triangular waveforms [26-28]. Figure 8 confirms that the initial Gaussian pulse reshapes into an almost rectangular pulse form at the point of optimum spectral compression, in agreement with the results presented in $[17,18]$. Compared to the input temporal waveform, the pulse is slightly shorter and has an approximately $20 \%$ higher peak power (in the lossless scenario being considered). As changes in the pulse shape bring about corresponding changes in the temporal gradient of the pulse profile, the SPM-induced frequency chirp tends to become linear and flat over most of the pulse. This contrasts with the purely nonlinear regime (Fig. 2(b)) in which the chirp is not compensated at the point of optimum compression. The TBP of the pulse is 0.86 , which is comparable to the transform limit for a rectangular pulse $(0.88)$. This expounds why the observed spectral profile (Fig. 5) is very close to the spectrum that would be obtained in the case of a perfect spectral compression (i.e., assuming a perfectly flat chirp profile). The nearly rectangular pulse shape also explains the train of oscillations that is observed in the compressed spectrum, which is typical of the cardinal sine shape of the Fourier transform of a rectangular waveform. Therefore, the spectral compression process in the presence of GVD provides a passive method for generating neartransform-limited pulses with a rectangular time envelope $[17,18]$. Note that the formed pulses differentiate from the rectangular temporal waveforms with a linear chirp variation over most of the pulse that correspond to the nonlinear evolution regime in a normally dispersive fiber immediately preceding wave breaking [24-26].
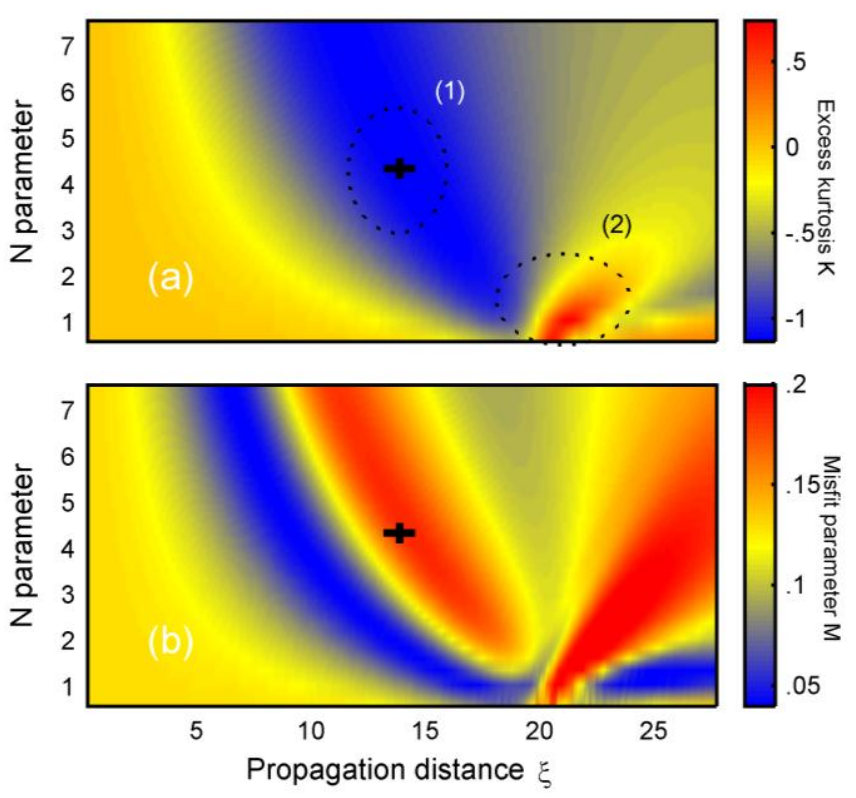

Fig. 7. Evolution of: (a) the excess kurtosis parameter and (b) the misfit parameter to a parabolic temporal shape versus $\xi$ and $N$ in a nonlinear dispersive fiber for an initial Gaussian pulse with the stretching factor $A=20$. The sign plus denotes the point of maximum Strehl ratio.

The evolution of the misfit parameter (Fig. 7(b)) reveals that the pulse acquires an almost parabolic intensity profile before the point of optimum spectral compression. As it can be seen from Fig. 8, the formed pulse features a very low chirp value over its central part. This is in strong contrast with the linearly chirped parabolic pulses that can be generated through progressive pulse reshaping upon nonlinear propagation in a normally dispersive fiber, which are accompanied by a significantly wider spectrum than that of the input pulse [22, 26, 29 , $30]$. Note also that the monotonic variation of the chirp across the pulse prevents oscillations from arising in the spectrum (Fig. 5).

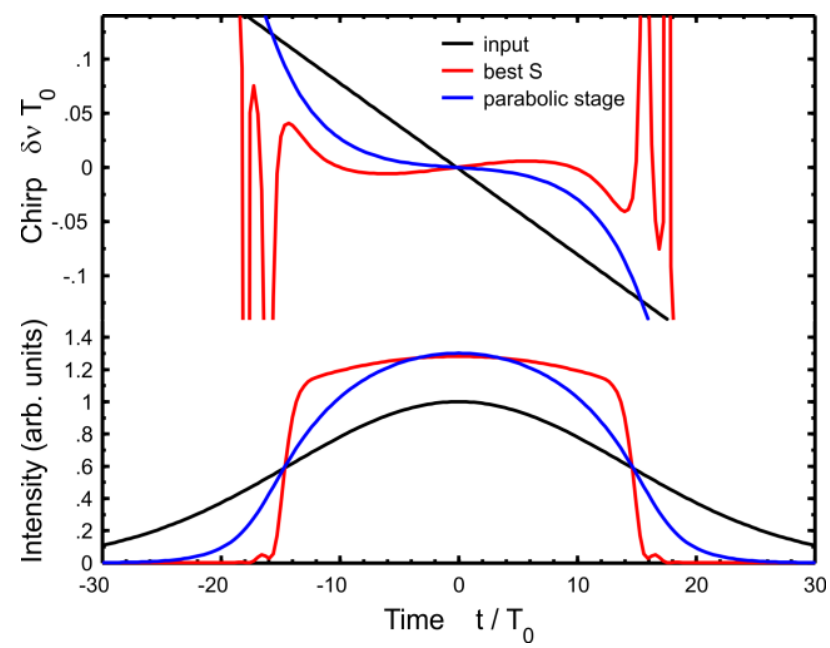

Fig. 8. Temporal intensity and chirp profiles of an initial Gaussian pulse with the stretching factor $A=20$ and the optimum power parameter $N_{\text {opt }}$ at: the entrance of the fiber (black), the propagation distance of optimum spectral compression (red), and the distance of reshaping into an almost parabolic pulse form (blue). 


\section{IMPACT OF INITIAL PULSE PROPERTIES}

We also studied the effect of the initial temporal broadening factor $A$ and pulse shape on the spectral compression that takes place at the optimum operational conditions defined by $\left(\xi_{\text {opt, }} N_{\text {opt }}\right)$. In Fig. 9 we summarize results for the evolution of $\xi_{\text {opt }}$ and $N_{\text {opt }}$ with $A$ for initial Gaussian and hyperbolic-secant pulses. It is seen that for both initial conditions the larger the initial stretching ratio, the higher the value of the optimum power parameter and the longer the optimum normalized propagation distance. Furthermore, optimum compression of the hyperbolic secant pulse requires smaller power parameter and longer normalized distance than that of the Gaussian pulse.

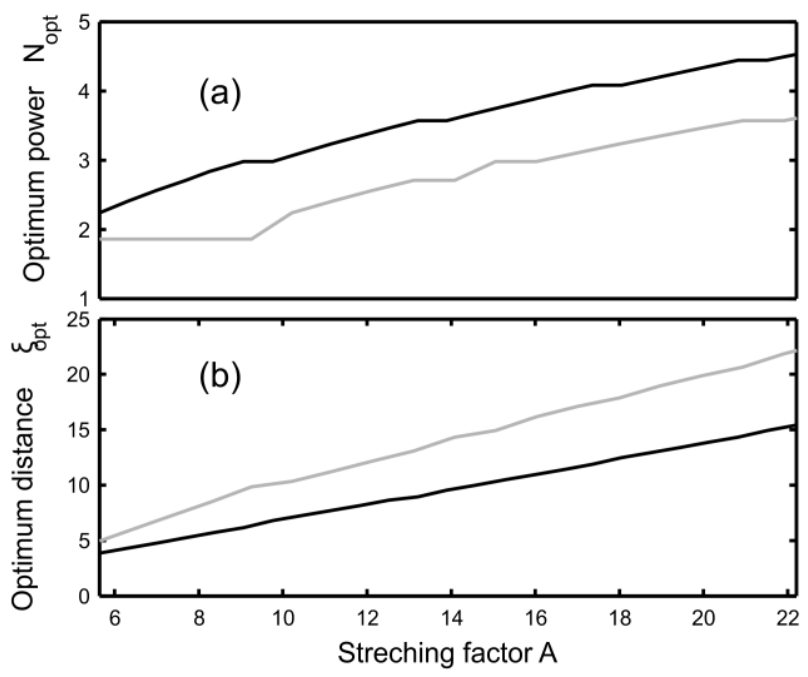

Fig. 9. Evolution of: (a) the optimum power parameter and (b) the optimum normalized propagation distance versus stretching factor $A$ for initial Gaussian (black) and hyperbolic-secant (grey) pulses.

The influence of the initial stretching factor on the performance characteristics of the optimum compression process is illustrated on Fig. 10. We can see from panel (a) that for all $A$ values, the spectral compression factor at the point of optimum compression is smaller than that obtained in the purely nonlinear regime. The Strehl ratio calculated using the actual temporal profile of the pulse at the optimum compression point (panel (b)) takes values above 0.9 , thus proving the nearly bandwidth-limited nature of the optimally compressed pulse. The GVD remarkably enhances the quality of the compression compared with the purely nonlinear regime. Moreover, contrarily to the pure nonlinear case, the degradation of the compression quality with increasing $A$ values is negligible. By contrast, if one is interested in the Strehl ratio defined in terms of the initial pulse profile, then the pinpointed compression quality is close to that of the pure SPM-driven case. The excess kurtosis plotted in panel (c) indicates that the flattening of the temporal profile of the pulse at the point of optimum compression is significant and almost independent of the stretching of the initial pulse. Figure 10 also shows overall outperformance of the initial Gaussian pulse over the hyperbolic-secant one.
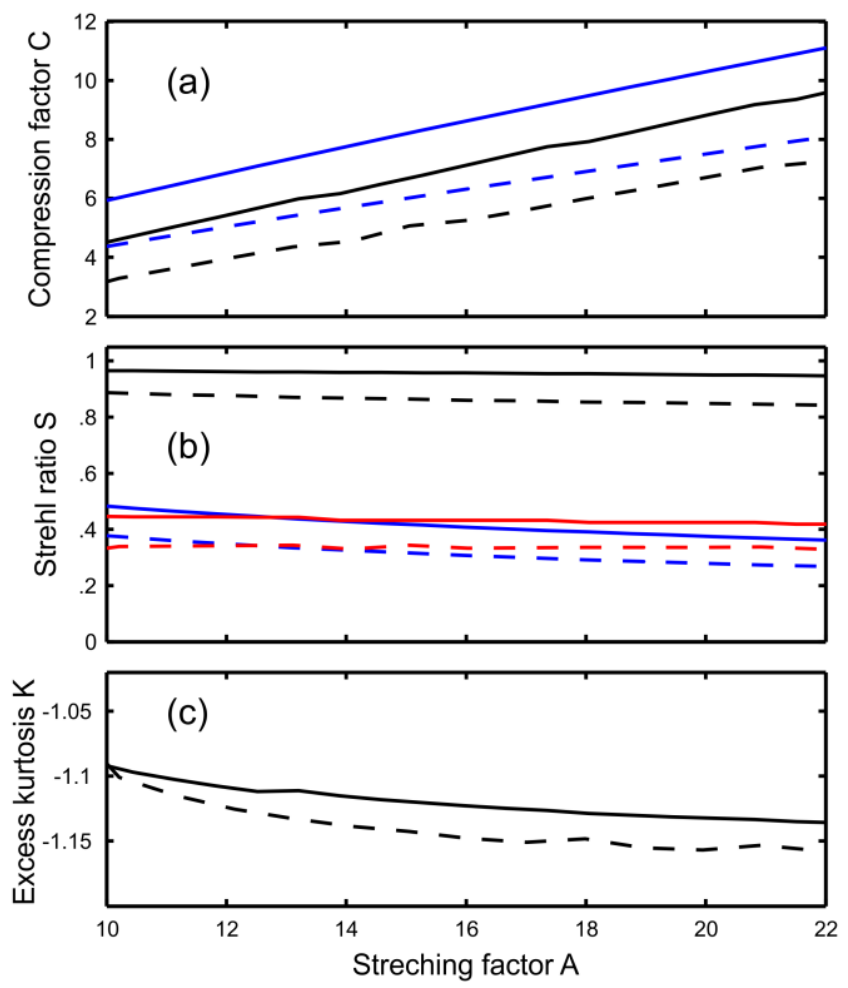

Fig. 10. Evolution of: (a) the spectral compression factor $C$, (b) the Strehl ratio $S$, and (c) the excess kurtosis parameter $K$ at ( $\xi_{\text {opt }} N_{\text {opt }}$ ) versus initial stretching ratio $A$ for initial Gaussian (solid) and hyperbolic-secant (dashed) pulses. Results are shown for the dispersive nonlinear (black) and the purely nonlinear (blue) regimes. Also shown is the Strehl ratio defined in terms of the initial pulse shape (red).

\section{CONCLUSION}

We have presented an in-depth characterization of the spectral compression of negatively chirped pulses that occurs upon propagation in a normally dispersive fiber. The study enables general rules for the design of spectral-compression fiber schemes over the experimentally accessible parameter space. We have shown that the GVD of the fiber plays a considerable role in the compression process and can enhance the quality of the compressed spectrum of the pulse. Indeed, the progressive reshaping of the pulse temporal profile upon dispersive nonlinear propagation helps increase the spectral Strehl ratio so as to achieve a near-bandwidth-limited spectrally compressed pulse. We have also shown that nearly parabolic and rectangular pulses with low levels of chirp are generated in the parameter region of optimum spectral compression.

A natural extension of this work would include the impact of optical gain on the spectral compression process [10] or the analysis of a multistage fiber amplifier comprising a spectral compression stage [31]. An accurate description of the resulting pulse dynamics could indeed provide a deeper insight into the rich dynamics occurring in fiber cavities [32, 33] or help a better understanding of the pulse spectrum narrowing induced by external phase modulators [34].

\section{Funding Information}

The authors acknowledge financial support from the PARI PHOTCOM Région Bourgogne and the Labex ACTION (ANR-11-LABX-01-01). 


\section{References}

1. R. H. Stolen, and C. Lin, "Self-phase modulation in silica optical fibers," Phys. Rev. A 17, 1448-1453 (1978).

2. N. Nishizawa, H. Takahashi, Y. Ozeki, and K. Itoh, "Wideband spectral compression of wavelength-tunable ultrashort soliton pulse using comb-profile fibre," Opt. Express 18, 11700-11706 (2010).

3. K. Tamura, and M. Nakazawa, "54-fs, $10-\mathrm{GHz}$ soliton generation from a polarization-maintaining dispersion-flattened dispersion-decreasing fiber pulse compressor," Opt. Lett. 26, 762-764 (2001).

4. A. B. Fedotov, A. A. Voronin, I. V. Fedotov, A. A. Ivanov, and A. M. Zheltikov, "Spectral compression of frequency-shifting solitons in a photonic-crystal fiber," Opt. Lett. 34, 662-664 (2009).

5. A. V. Zohrabian, and L. K. Mouradian, "Compression of the spectrum of picosecond ultrashort pulses," Quantum Electronics 25, 1076 (1995).

6. M. Oberthaler, and R. A. Höpfel, "Spectral narrowing of ultrashort laser pulses by self-phase modulation in optical fibers," Appl. Phys. Lett. 63, 1017-1019 (1993).

7. S. A. Planas, N. L. Pires Mansur, C. H. Brito Cruz, and H. L. Fragnito, "Spectral narrowing in the propagation of chirped pulses in single-mode fibers," Opt. Lett. 18, 699-701 (1993).

8. B. R. Washburn, J. A. Buck, and S. E. Ralph, "Transform-limited spectral compression due to self-phase modulation in fibers," Opt. Lett. 25, 445-447 (2000).

9. J. P. Limpert, T. Gabler, A. Liem, H. Zellmer, and A. Tünnermann, "SPMinduced spectral compression of picosecond pulses in a single-mode Yb-doped fiber amplifier," Appl. Phys. B 74, 191-195 (2002).

10. J. P. Limpert, A. Liem, T. Gabler, H. Zellmer, A. Tünnermann, S. Unger, S. Jetschke, and H.-R. Müller, "High-average-power picosecond Yb-doped fiber amplifier," Opt. Lett. 16, 1849-1851 (2001).

11. M. Rusu, and O. G. Okhotnikov, "All-fiber picosecond laser source based on nonlinear spectral compression," Appl. Phys. Lett. 89, 091118 (2006).

12. C. Bao, X. Xiao, and C. Yang, "Spectral compression of a dispersion-managed mode-locked Tm:fiber laser at 1.9 um," IEEE Photon. Technol. Lett. in press (2015).

13. E. R. Andresen, J. Thogersen, and S. R. Keiding, "Spectral compression of femtosecond pulses in photonic crystal fibers," Opt. Lett. 30, 2025-2027 (2005).

14. D. A. Sidorov-Biryukov, A. Fernandez, L. Zhu, A. Pugzlys, E. E. Serebryannikov, A. Baltuska, and A. M. Zheltikov, "Spectral narrowing of chirp-free light pulses in anomalously dispersive, highly nonlinear photonic-crystal fibers," Opt. Express 16, 2502-2507 (2008).

15. E. R. Andresen, J. M. Dudley, C. Finot, D. Oron, and H. Rigneault, "Transformlimited spectral compression by self-phase modulation of amplitude shaped pulses with negative chirp," Opt. Lett. 36, 707-709 (2011).

16. J. Fatome, B. Kibler, E. R. Andresen, H. Rigneault, and C. Finot, "All-fiber spectral compression of picosecond pulses at telecommunication wavelength enhanced by amplitude shaping," Appl. Opt. 51, 4547-4553 (2012).

17. A. A. Kutuzyan, T. G. Mansuryan, G. L. Esayan, R. S. Akobyan, and L. K. Mouradian, "Dispersive regime of spectral compression," Quantum Electronics 38, 383-387 (2008)

18. M. A. Kalashyan, K. A. Palandzhyan, G. L. Esayan, and L. K. Muradyan, "Generation of transform-limited rectangular pulses in a spectral compressor," Quantum Electronics 40, 868 (2010).

19. G. P. Agrawal, Nonlinear Fiber Optics, Fourth Edition (Academic Press, San Francisco, CA, 2006).

20. L. T. DeCarlo, "On the meaning and use of kurtosis," Psychol. Methods 2, 292 307 (1997).

21. A. Ruehl, O. Prochnow, D. Wandt, D. Kracht, B. Burgoyne, N. Godbout, and S. Lacroix, "Dynamics of parabolic pulses in an ultrafast fiber laser," Opt. Lett. 31, 27342736 (2006).

22. C. Finot, L. Provost, P. Petropoulos, and D. J. Richardson, "Parabolic pulse generation through passive nonlinear pulse reshaping in a normally dispersive two segment fiber device," Opt. Express 15, 852-864 (2007).

23. M. D. Perry, T. Ditmire, and B. C. Stuart, "Self-phase modulation in chirpedpulse amplification," Opt. Lett. 19, 2149-2151 (1994).

24. D. Anderson, M. Desaix, M. Lisak, and M. L. Quiroga-Teixeiro, "Wave-breaking in nonlinear optical fibers," J. Opt. Soc. Am. B 9, 1358-1361 (1992).

25. C. Finot, B. Kibler, L. Provost, and S. Wabnitz, "Beneficial impact of wavebreaking on coherent continuum formation in normally dispersive nonlinear fibers," J. Opt. Soc. Am. B 25, 1938-1948 (2008).

26. S. Boscolo, A. I. Latkin, and S. K. Turitsyn, "Passive nonlinear pulse shaping in normally dispersive fiber systems," IEEE J. Quantum Electron. 44, 1196-1203 (2008).

27. N. Verscheure, and C. Finot, "Pulse doubling and wavelength conversion through triangular nonlinear pulse reshaping," Electron. Lett. 47, 1194-1196 (2011).
28. S. O. lakushev, O. V. Shulika, I. A. Sukhoivanov, V. I. Fesenko, M. V. Andrés, and H. Sayinc, "Formation of ultrashort triangular pulses in optical fibers," Optics Express 22, 29119-29134 (2014).

29. C. Finot, J. M. Dudley, B. Kibler, D. J. Richardson, and G. Millot, "Optical parabolic pulse generation and applications," IEEE J. Quantum Electron. 45, 1482 1489 (2009).

30. S. O. lakushev, O. V. Shulika, and I. A. Sukhoivanov, "Passive nonlinear reshaping towards parabolic pulses in the steady-state regime in optical fibers," Opt. Commun. 285, 4493-4499 (2012).

31. D. A. Korobko, O. G. Okhotnikov, and I. O. Zolotovskii, "Multistage fiber preamplifier comprising spectral compression for generation of high-energy laser pulses "J. Opt. Soc. Am. B in press.

32. S. Boscolo, and S. K. Turitsyn, "Intermediate asymptotics in nonlinear optical systems," Phys. Rev. A 85, 043811 (2012).

33. S. Boscolo, S. K. Turitsyn, and C. Finot, "Amplifier similariton fiber laser with nonlinear spectral compression," Opt. Lett. 37, 4531-4533 (2012).

34. B. W. Plansinis, W. R. Donaldson, and G. P. Agrawal, "Spectral changes induced by a phase modulator acting as a time lens," J. Opt. Soc. Am. B 32, 1550-1554 (2015). 